\title{
A Medical Mobile Application For Managing A Heart And Lung Machine During Cardiac Surgery
}

Prarinya Boonchai

Naresuan University

\section{Supaporn Kulthinee}

Chulabhorn International College of Medicine, Thammasat University

Phatiwat Chotimol ( $\sim$ phatiwatch@nu.ac.th )

Naresuan University

\section{Research Article}

Keywords: mobile application, managing, heart and lung machine

Posted Date: May 26th, 2021

DOl: https://doi.org/10.21203/rs.3.rs-509048/v1

License: (1) This work is licensed under a Creative Commons Attribution 4.0 International License.

Read Full License 


\section{Abstract}

Background: Opened heart surgery with cardiopulmonary bypass (CPB) is a critical and complex procedure. A Heart-Lung machine (HLM) plays an important role for controlling the cardiopulmonary functions during the time of the surgery. Perfusionist must consider a variety of essential factors and calculate several cardiovascular parameters regarding the process of operating a HLM. To improving the quality of work, personal digital assistants must continually develop their skills and knowledge levels.

Objective: The goal of this work is to construct a mobile application device that has a wide variety of functions which has the capacity to control targeted clinical planning and decision making for HLM users so to enable them to have control and evaluate the mobile application to user's satisfaction.

Methods: This smartphone app was constructed base on the ionic framework. The researchers have developed an unique algorithms for operating the HLM. The app was generated according to the phase of design, algorithm, validation, and user's satisfaction of perfusionists.

Results: The Project Researchers have officially assigned this medical mobile application with the name is Perfusion Assistant app that can be accessed and used effectively cross platform on iOS and Android. The application is comprised of five main categories which includes: a perfusion calculator, myocardial protection chart, drugs details, priming solution and parameters values. Result shown that all cardiovascular parameters did not significant differ from Perfusion Assistant app when compared to manual calculation. User's satisfaction was at $3.64 \pm 0.76$ in the first evaluation. After modification with feedback from experts, the app was evaluated with a $4.13 \pm 0.56$ satisfaction.

Conclusions: Perfusion Assistant app is an application designed in clinical planning and decision of HLM controlling for perfusionists and medical staff that work in an opened heart surgery arena. Perfusion Assistant app offers a variety of calculations related to CPB including blood flow rate, systemic vascular resistant, priming volume, and predicted hematocrit. Furthermore, Perfusion Assistant app provides a quick, easy access, and real-time application for CPB that user's satisfaction was a good level.

\section{Introduction}

Since John Gibbon construct and develop heart and lung machine (HLM) for cardiopulmonary bypass (CPB), this machine and principal has been used in opened heart surgery over 60 years [1]. HLM controlled by perfusinists mimics the cardiopulmonary function by temporarily taking over their functions during cardiac surgery [2]. However, perfusinists are still required to attentive regarding the monitoring of the blood volume in a venous reservoir of an oxygenator, the mean arterial blood pressures (MAP), and the systemic vascular resistance (SVR) continuously in order to adjust and maintain the perfusion blood flow rate (BFR) throughout a centrifugal or roller pump in a CPB circuit [3]. If perfusionists can be accessed automatically by way of the mobile app for a planned operation, it will be patients' safety; for example: the App can be used to calculate optimal blood flow, SVR, priming volume, and provide a hematocrit prediction in order to reduce post-operative complications. Furthermore, regulation of pump 
flow rate dependent SVR to ensure that pump flow is adequate [4]. Moreover, perfusionist need to be considered and calculated into the open heart surgery procedure several cardiovascular parameters for use of a HLM. Opened heart surgery with CPB is a critical and complex procedure; so, to improve the quality of work, personal digital assistants should to develop in order to help operating room staffs and perfusionists to provide a more accurate system for treating patients.

Mobile applications have been able to help improve one's efficiency and knowledgebase when faced with challenges. They are fundamental benefits that make life easier and help streamline job responsibilities. Moreover, the advantages of a medical mobile application improve access to medical literature and clinical work plan [5-7]. Another contribution of this work is that previous studies have shown that mobile application on smartphones on iOS and Android are popularly used among physicians and professional medical staffs for the past decade [8]. Therefore, the aim of this work is to construct a mobile application device that offers a wide variety of functions that include: bypass flow rate, SVR, priming volume, and predicted hematocrit, which can assist in perfusionist decision making regarding HLM controlling and then to evaluation user's satisfaction.

\section{Methods}

This smartphone app was constructed and developed base on an ionic framework. (ionicframework, AngularJS, Cordova, HTML5, JavaScript), which are unique algorithms used in the operation of HLM. The app was constructed and developed utilizing the following phases: design, algorithm, validation, and evaluation user's satisfaction. Overall, the Perfusion Assistance system architecture platforms are as shown in Figure 1.

\section{Mobile application design phase}

The scope of the discussion process to create a workable HLM application was daunting with principle of cardiopulmonary bypass [9]. (Figure 2) The researchers were able to go through a brainstorming to determine the steps necessary to fill the knowledge gap that would make the HLM process more reliable and practical for perfusionists and operating room staffs.

This medical mobile application which is referred to as the Perfusion Assistant app. has five typically categories of concerns that most perfusionists are interested when using an application programs that relates toothier professional responsibilities. Application functions such as perfusion calculator, myocardial protection, drugs, priming solution, parameters values, location and about us icon. (Figure 3 )

\section{Algorithm mobile application phase}

The algorithm phase is used to identify the multiple concepts, steps, and application's flow mapping. During this phase, the programmer converts the appropriate and complex data and information into an algorithm. Once the formatting of the basic algorithms was complete, 
we to evaluate mobile application by cardiothoracic surgeons and perfusionists. Afterwards, comments were reviewed and the suggestions were received on how to improve the unique algorithms. The researchers then reconstruct the arrays into the final Perfusion Assistant app. For example, the unique algorithm used for the perfusion calculator are shown in Figures 4.

\section{Validation mobile application phase}

After development mobile application, the accuracy of Perfusion Assistant app final version was examined. The medical information contents were reviewed by five professional perfusionists and five cardiothoracic surgeons. Moreover, the completeness of medical contents was checked for any inconsistencies or mistakes in the text; in addition, the language format and usage in the applications were edited and corrected by a native English language expertise at the Language Center, Naresuan University (LCNU). Every aspect of the perfusion calculator formats and formulas received approval.

\section{Evaluation user's satisfaction phase.}

After validation completion, a team of experts were assembled. To evaluate application user's satisfaction, ten perfusinists were asked to use the the new revision of the "Perfusion Assistant app." and provide the researchers with an updated evaluation. Afterwards, the level of satisfaction of ten perfusionists in second evaluation was examined. The application user's satisfaction questionnaire was scored based on a 5-point Likert scale.

\section{Statistical Analysis}

Descriptive data was expressed as mean \pm standard deviation (SD), frequencies, or as an absolute number and percentages when appropriate. The SPSS software version 17 (SPSS Inc, Chicago, IL, USA) was used for all of our analysis. $P$ values $<0.05$ were statistically significant.

\section{Results}

We were able to construct and develop the medical mobile app to provide the Perfusionist a more suitable working load. The Perfusion Assistant app, an mobile application, can be accessed effectively cross platform both on iOS and Android. This smartphone app was designed to be easy to use. Tapping the startup screen makes the main menu appear; once the startup screen disappears, the five main sections which are presented by icons, perfusion calculator, drugs, myocardial protection, priming solution, and parameters value appear, on the screen. The startup screen and home menu, along with the contents of the main menu are shown in Figs. 2.

The result illustrated that $\mathrm{BSA}, \mathrm{BFR}$, predicted $\mathrm{Hct}, \mathrm{SVR}, \mathrm{CaO}_{2}, \mathrm{CvO}_{2}, \mathrm{DO}_{2}$ and $\mathrm{VO}_{2}$ did not significant differ from Perfusion Assistant app when compared to manual calculations. The variables related to the perfusion data collection and analysis of data between manual format and the Perfusion Assistant app are presented in Table 1. 
Table 1

Manual compared to Perfusion Assistant app data calculations per variable.

\begin{tabular}{|llll|}
\hline Variable & Manual & Perfusion Assistant app. & p - value \\
\hline $\mathrm{BSA}$ & $1.65 \pm 0.075$ & $1.65 \pm 0.075$ & 0.083 \\
\hline Minimum BFR & $3.297 \pm 0.150$ & $3.299 \pm 0.150$ & 0.093 \\
\hline Optimum BFR & $3.958 \pm 0.181$ & $3.959 \pm 0.181$ & 0.065 \\
\hline Maximum BFR & $4.947 \pm 0.226$ & $4.949 \pm 0.226$ & 0.223 \\
\hline Predicted Hct & $31.206 \pm 0.997$ & $31.206 \pm 0.997$ & 0.651 \\
\hline $\mathrm{SVR}$ & $1,323.520 \pm 45.970$ & $1,323.52 \pm 45.970$ & 0.157 \\
\hline $\mathrm{CaO}$ & $16.840 \pm 0.320$ & $16.840 \pm 0.320$ & 0.404 \\
\hline $\mathrm{CVO}_{2}$ & $12.690 \pm 0.260$ & $12.690 \pm 0.260$ & 0.317 \\
\hline $\mathrm{DO}_{2}$ & $663.841 \pm 18.689$ & $663.841 \pm 18.689$ & 1.00 \\
\hline $\mathrm{VO}_{2}$ & $157.984 \pm 8.199$ & $157.984 \pm 8.199$ & 0.317 \\
\hline
\end{tabular}

To evaluate on Perfusion Assistant mobile application users' satisfaction, 20 perfusionists were asked to use the app and then assess and provide their personal level of satisfaction so that the researchers are able to identify the appropriateness and usefulness of the app. All perfusionists were members of the Society of Cardio Thoracic Technologist of Thailand. The mean age of users was 34.16 years (range, 26 - 63 years); 5 (25\%) were male and 15 (25\%) were female; 95\% were cardiothoracic technologist that are practicing perfusionists, while (85\%) were bachelor degree, and (15\%) were master degree. The mean regarding the duration of work as a perfusionist was 9.83 years as shown in Table 2. 
Table 2

The characteristics of participants.

\begin{tabular}{|ll|}
\hline Characteristics of participants & Value $(\mathbf{n}=\mathbf{2 0})$ \\
\hline Age (years) & $34.16(26-63)$ \\
\hline Male, \% (n) & $25 \%(5)$ \\
\hline Duration of work as perfusionist (years) & 9.83 \\
\hline Major academic & \\
Cardiothoracic technology \% (n) & $95 \%(19)$ \\
Nursing \% (n) & $5 \%(1)$ \\
\hline $\begin{array}{l}\text { Study level } \\
\text { Bachelor degree \% (n) } \\
\text { Master degree \% (n) }\end{array}$ & $85 \%(17)$ \\
\hline
\end{tabular}

User's satisfaction was at $3.64 \pm 0.76$ in the first evaluation. Based on their feedback, the app required slightly modification. User's satisfaction was at a good level; however, in the second evaluation the application performed at a higher level, $4.13 \pm 0.56$. Nevertheless, based on user's comments, the programmer modified the string of arrays used in the app. The mean values of user's satisfaction according to the data presented in the ten items listed in the questionnaire are shown in Table 3. 
Table 3

User's satisfaction on "Perfusion Assistant" mobile application.

\begin{tabular}{|llll|}
\hline Category & $\begin{array}{l}\text { First } \\
\text { evaluation } \\
(\mathbf{n = 1 0})\end{array}$ & $\begin{array}{l}\text { Second } \\
\text { evaluation } \\
(\mathbf{n}=10)\end{array}$ & $\begin{array}{l}\text { p- } \\
\text { value }\end{array}$ \\
\hline 1. Usability & $3.90 \pm 0.56$ & $4.05 \pm 0.60$ & 0.403 \\
\hline 2. Designed to be user-friendly & $3.60 \pm 0.84$ & $4.20 \pm 0.52$ & 0.841 \\
\hline 3. Content is properly related to design objectives & $4.00 \pm 0.81$ & $4.25 \pm 0.44$ & 0.398 \\
\hline 4. Provides clarity of content usage & $3.40 \pm 0.51$ & $4.20 \pm 0.52$ & 0.598 \\
\hline 5. Prevents potential faults & $3.10 \pm 0.73$ & $3.70 \pm 0.73$ & 0.085 \\
\hline 6. The processing speed of the application & $3.90 \pm 0.87$ & $4.35 \pm 0.48$ & 0.572 \\
\hline 7. The reliability of the application & $3.50 \pm 0.84$ & $4.05 \pm 0.51$ & 0.585 \\
\hline $\begin{array}{l}\text { 8. Font size, design, and screen color are } \\
\text { appropriate. }\end{array}$ & $3.70 \pm 1.05$ & $4.10 \pm 0.64$ & 0.264 \\
\hline 9. Language usage & $3.60 \pm 0.51$ & $4.15 \pm 0.58$ & 0.435 \\
\hline 10. Appropriateness of screen size & $3.70 \pm 0.82$ & $4.25 \pm 0.55$ & 0.414 \\
\hline Average Mean \pm S.D. & $3.64 \pm 0.76$ & $4.13 \pm 0.56$ & 0.401 \\
\hline Value are showed as mean \pm Standard deviation & & & \\
\hline Five-point Likert scale used & & & \\
\hline
\end{tabular}

\section{Discussion}

Our Perfusion Assistant app is written in JavaScript, using ionic framework version 2 for the mobile application design and can be used on any mobile devices (phones or tablets) that use an Android and iOS platform [10]. The most challenging phase of the process of developing the clinical planning and decision regarding the HLM controlling smartphone app was designing the scope and algorithm for the mobile app. In this study, the scope of function and algorithm link were determined in collaborations with all involved researchers. Furthermore, we edited and developed of scope and algorithm based on suggestion from experts who participated in the first evaluation. In the first Perfusion Assistant app design, there were seven categories, which also included a blood gas interpretation. However, blood gas interpretations for this study require the storage of a much larger amount of contents; consequently, the configuration of the algorithms caused the program to be was not unstable once engaged, for smartphones possess an insufficient capability to store large amounts of contents. In addition, some specifications in blood gas function are not able to interpretation the data correctly. Therefore, we decide to temporarily remove the blood gas interpretation from this app. Nevertheless, blood gas interpretation is 
considered a very important variable for HLM controlling [11]; however, the perfusionist who have used the app have provided us with suggestions that will require additional development of the blood gas interpretation mode. Therefore, blood gas interpretation mode should to be further developed

The perfusion calculator mode has three sub mode which include: BFR, SVR, and oxygen content and consumption. The Mosteller equation, $\mathrm{BSA}=\frac{\sqrt{B W \cdot x H t}}{3600}$ [12] regarding body surface area, was applied to determine the following validation of BSA value when comparing the manual calculations with formulas and this mobile app. showed no significant difference. Concerning myocardial protection, the priming solution and the parameter values mode were for learning content only. Therefore, this app has grabbed the user' attention and to motivate learners. This work should have contained diverse videos as well as many photos and pictures. However, the researchers did not include videos in the app; for according to the app producer, video downloads on a smartphone require a large capacity memory. Moreover, app development can be a difficult process as a result of limited funds and the amount of technological support.

The regulation of BFR dependent MAP and SVR to ensure that pump flow is adequate [13]. For example, once patients obtain low MAP and low SVR, perfusionists will be able to increase arterial pump flow rate in HLM circuit to maintain an adequate tissue perfusion. Hence, the ability and a necessity of the applications were making a decision regarding the adjustment of the arterial pump flow rate by perfusionists.

The user satisfaction survey of the "Perfusion Assistant" app revealed good-quality subjective level of satisfaction. They showed a high satisfaction with the design, content, and usability of the program; therefore, there is a high anticipation that perfusionists will use this app for planning and making decision to assist in controlling HLM during opened heart surgery. Furthermore, this smartphone app educationally for cardiothoracic surgeons, anesthesiologist, scrub nurse, and medical staff. However, only a small number of participated in this survey. They suggested that having full knowledge of principle of CPB; therefore, there is a need for future work aimed add and focusing in detail on the learning content.

Several developed countries are making efforts for providing improved a semi-automated or automated circulation system for a HLM $[14,15]$. We hope as part of algorithm mobile application in this work may are fundamental data for biomedical engineer to develop automatic control of the HLM in next step of opened heart surgery arena.

\section{Conclusion}

Perfusion Assistant app is an application designed in clinical planning and decision of HLM controlling for perfusionists and medical staffs that work in the open heart surgery arena. Perfusion Assistant app offers variety of calculations related to cardiopulmonary bypass including BFR, SVR, priming volume and predicted hematocrit, Furthermore, this mobile app provides quick, easy access and real-time to the knowledge of cardiopulmonary bypass that user's satisfaction was a good level. 


\section{Abbreviations}

CPB: Cardiopulmonary bypass; HLM: Heart-lung machine; LCNU: Language center Naresuan university; SD: Standard deviation; BSA: Body surface area; BFR: Blood flow rate; Hct: Hematocrit; SVR: Systemic vascular resistance; $\mathrm{CaO}_{2}$ : Arterial oxygen content; $\mathrm{CvO}_{2}$ : Mixed venous oxygen content; $\mathrm{DO}_{2}$ : Oxygen delivery; $\mathrm{VO}_{2}$ oxygen consumption; $\mathrm{Ht}$ : Height; $\mathrm{Wt}$ : Weight; $\mathrm{Cl}$ : Cardiac index; $\mathrm{C}$ : concentration; $\mathrm{V}$ : volume

\section{Declarations}

\section{Ethics approval and consent to participate}

All subjects were informed of the aim of the study and gave their written consent form to participate. This study was approved by the Naresuan University ethics committee, approval number 293/2019.

\section{Consent for publication}

No application

\section{Availability of data and material}

The data generated during the current study are available from the corresponding author on reasonable request.

\section{Competing interests}

The authors have no conflicts to declare.

\section{Funding}

None

\section{Authors' contributions}

Design of experiments, evaluation and discussion of results, P.B., S.K.; Advising on all research, design of experiments, evaluation and discussion of results, and writing the manuscript, P.C. All authors read and approved the final manuscript.

\section{Acknowledgements}

We would like to thank Mr. Gregory Alan Smith, at the Language Center, Naresuan University(LCNU), for editing and correcting our manuscript.

\section{References}


[1]. Silvay G, Castillo JG. John Heysham Gibbon and the $60^{\text {th }}$ Anniversary of the First Successful HeartLung Machine: Brief Notes about the Development of Cardiac Surgery in Europe and Slovakia. Bratisl Med J. 2013;114(5):247-50.

[2]. Stammers AH, L BM, E AT, Coley T. Does Standardizing Extracorporeal Circuit Design for Cardiopulmonary Bypass Affect Outcomes? Results from a National Perfusion Registry. The journal of extra-corporeal technology. 2019;51(4):210-20.

[3]. Krishnamoorthy, B. (Ed.). (2020). Cardiothoracic Manual for Perioperative Practitioners. M\&K Update Ltd.

[4]. Kam PC, Hines L, O'Connor E. Effects of cardiopulmonary bypass on systemic vascular resistance. Perfusion. 1996;11(4):346-50.

[5]. Payne KFB, Wharrad H, Watts K. Smartphone and medical related App use among medical students and junior doctors in the United Kingdom (UK): a regional survey. Bmc Med Inform Decis. 2012;12.

[6]. O'Connor P, Byrne D, Butt M, Offiah G, Lydon S, Mc Inerney K, et al. Interns and their smartphones: use for clinical practice. Postgrad Med J. 2014;90(1060):75-9.

[7]. Hardyman W, Bullock A, Brown A, Carter-Ingram S, et al. Mobile technology supporting trainee doctors' workplace learning and patient care: an evaluation. BMC medical education. 2013;13(1), 6.

[8]. Boruff JT, Storie D. Mobile devices in medicine: a survey of how medical students, residents, and faculty use smartphones and other mobile devices to find information. J Med Libr Assoc. 2014;102(1):2230 .

[9]. Gravlee GP, Davis RF, Stammers AH, Ungerleider, RM. Cardiopulmonary Bypass: Principles and Practice. 3 rd ed. Philadelphia: Wolters Kluwer/Lippincott Williams and Wilkins; 2007.

[10]. Waranashiwar J, \& Ukey M. Ionic Framework with Angular for Hybrid App Development. International Journal of New Technology and Research. 2018;4(5), 263068.

[11]. Sanjay OP, Devnath A, Thejas BC. Arterial blood gas management during cardiopulmonary bypass. Indian journal of clinical biochemistry : IJCB. 2005;20(1):98-102.

[12]. Mosteller, R. D.\&quot;Simplified calculation of body-surface area.\&quot; N Engl J Med. 1987;317(17): 1098.

[13]. Murphy GS, Hessel EA, 2nd, Groom RC. Optimal perfusion during cardiopulmonary bypass: an evidence-based approach. Anesthesia and analgesia. 2009;108(5):1394-417.

[14]. Birnbaum D, Philipp A, Kaluza M, Detterbeck M.[On the way to an automatic heart-lung machine: a control system for oxygen tension in the oxygenator]. Biomedizinische Technik Biomedical engineering. 
1997;42 Suppl: 313 - 4.

[15]. Beyersdorf F. New dimensions for extracorporeal circulation. Interactive cardiovascular and thoracic surgery. 2017;24(4):479-81.

\section{Figures}

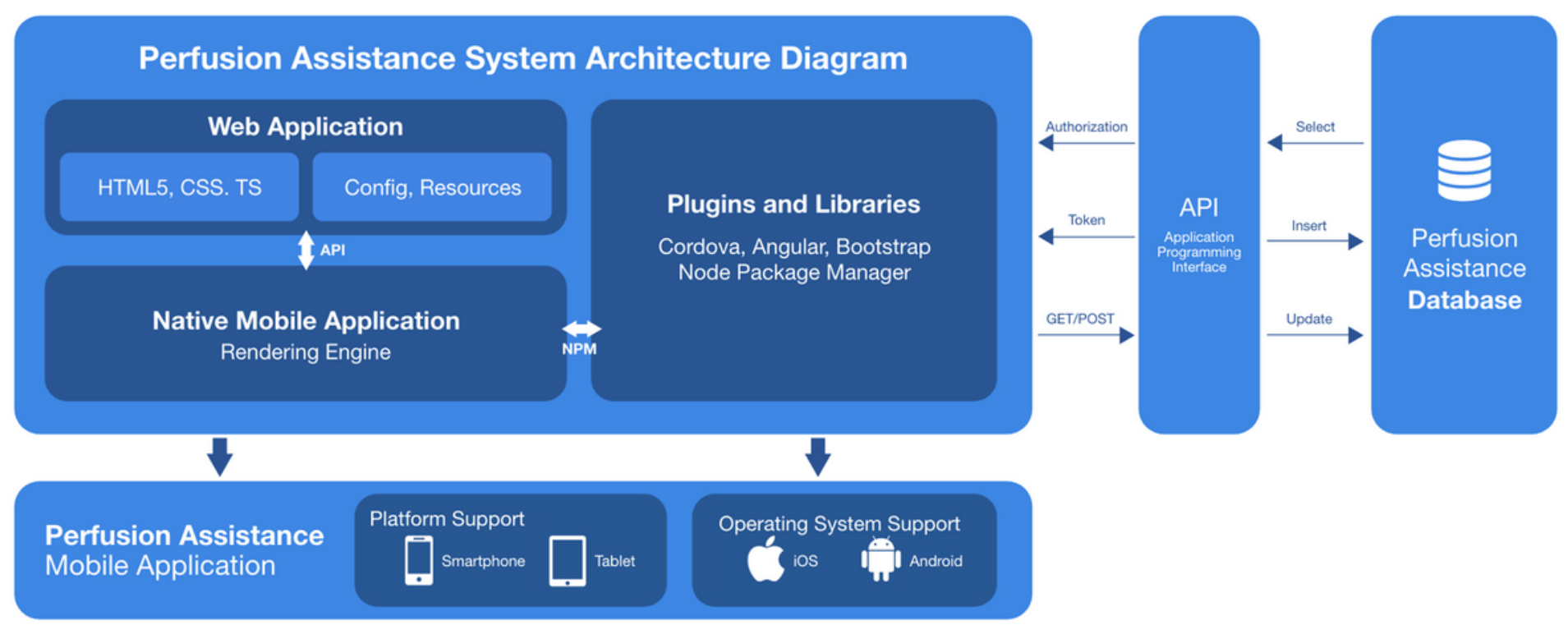

\section{Figure 1}

Assistance system architecture diagram 


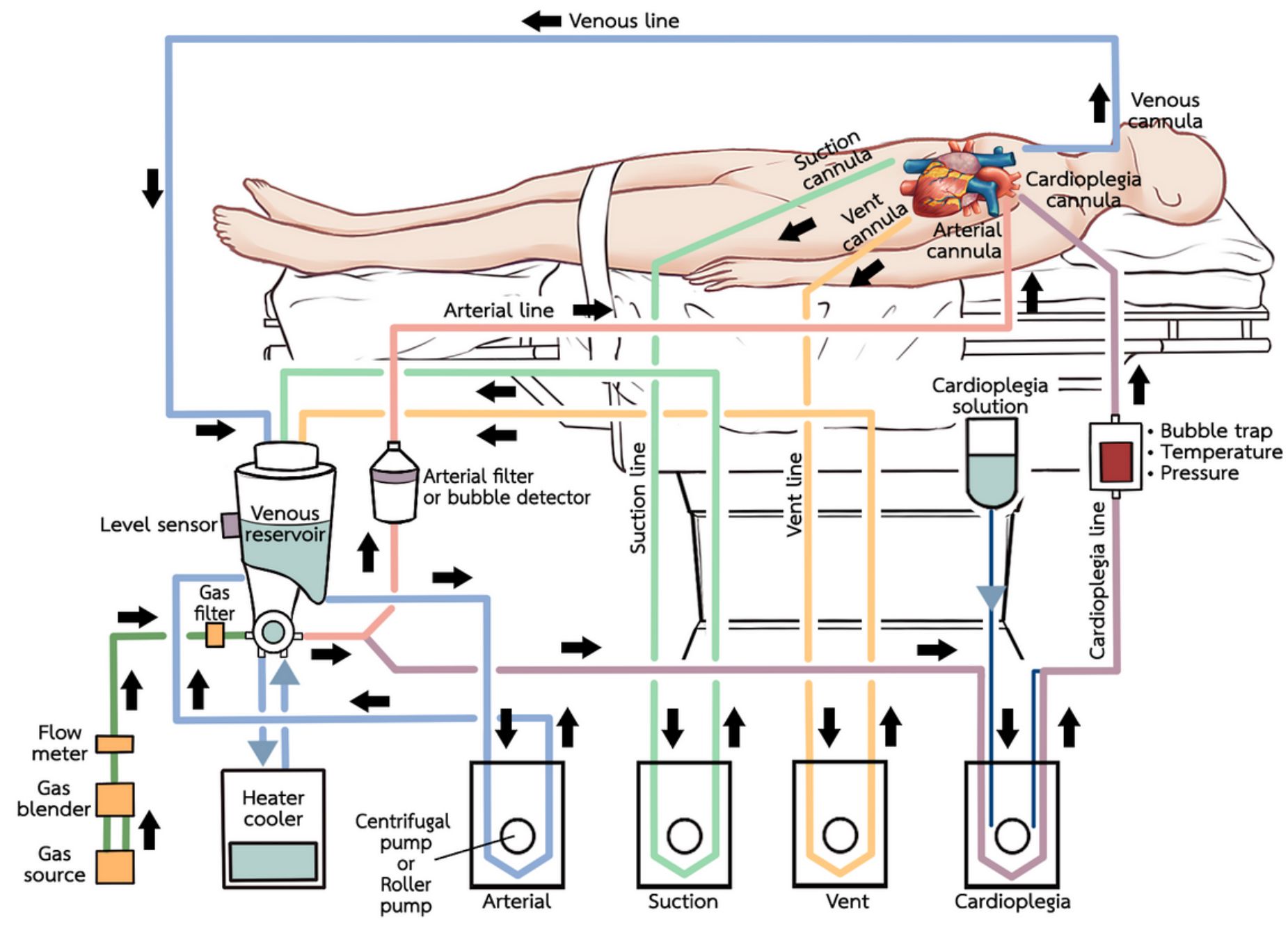

Figure 2

The fundamental understanding of cardiopulmonary bypass through the use of a heart and lung machine 


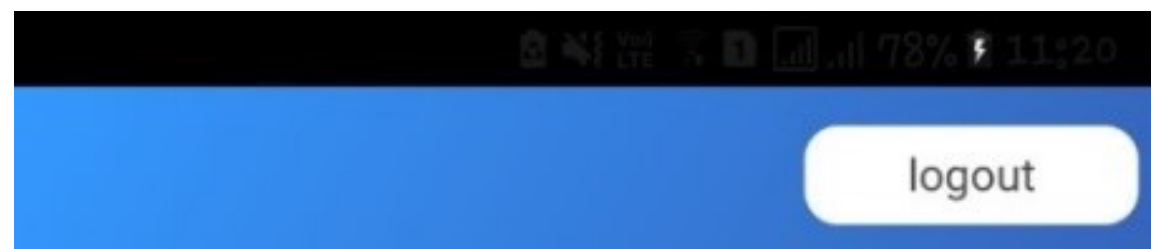

\section{Welcome to}

\section{Perfusion Assistant}

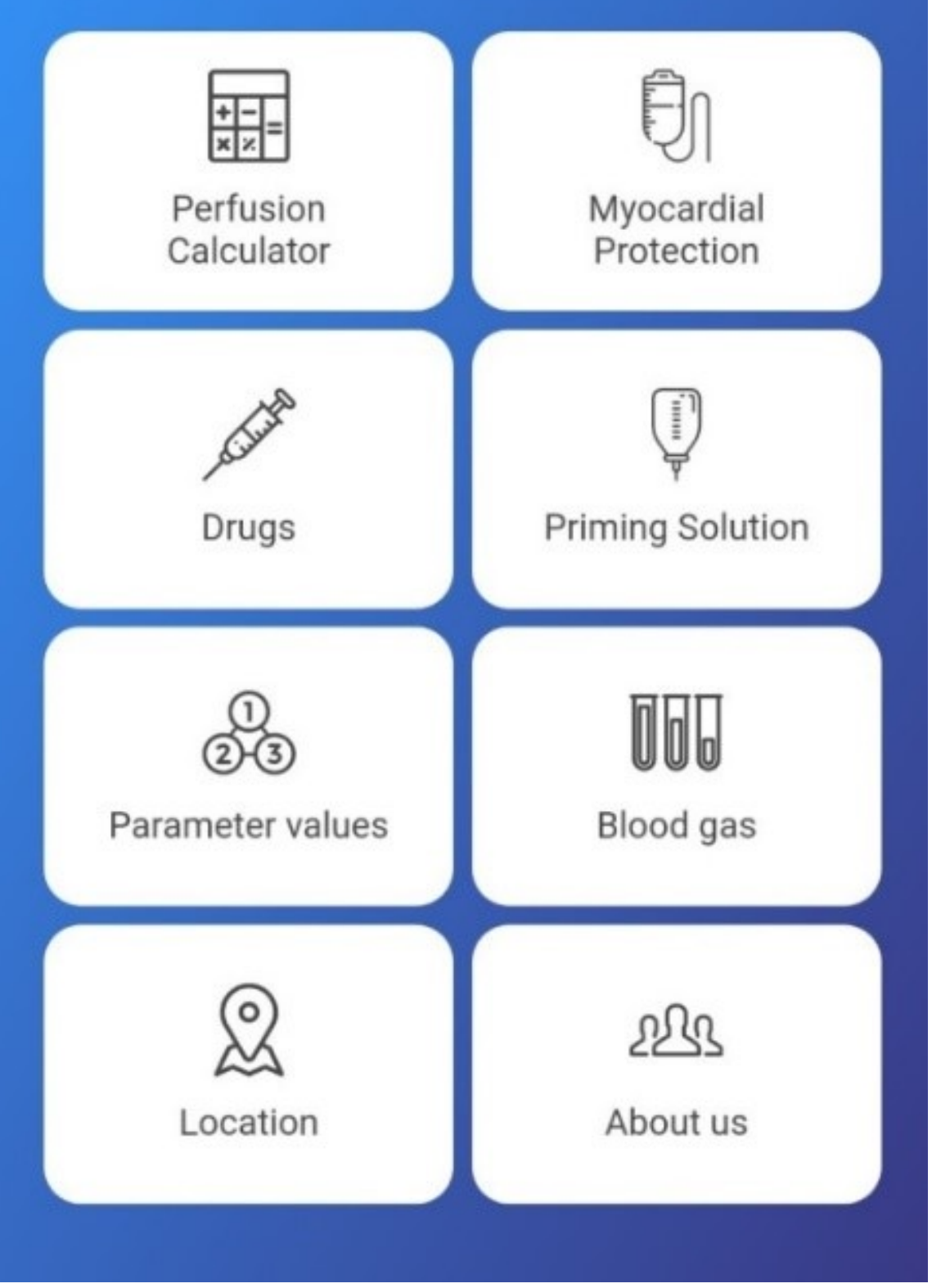

Figure 3

Main and additional information icon of Perfusion Assistant app. 


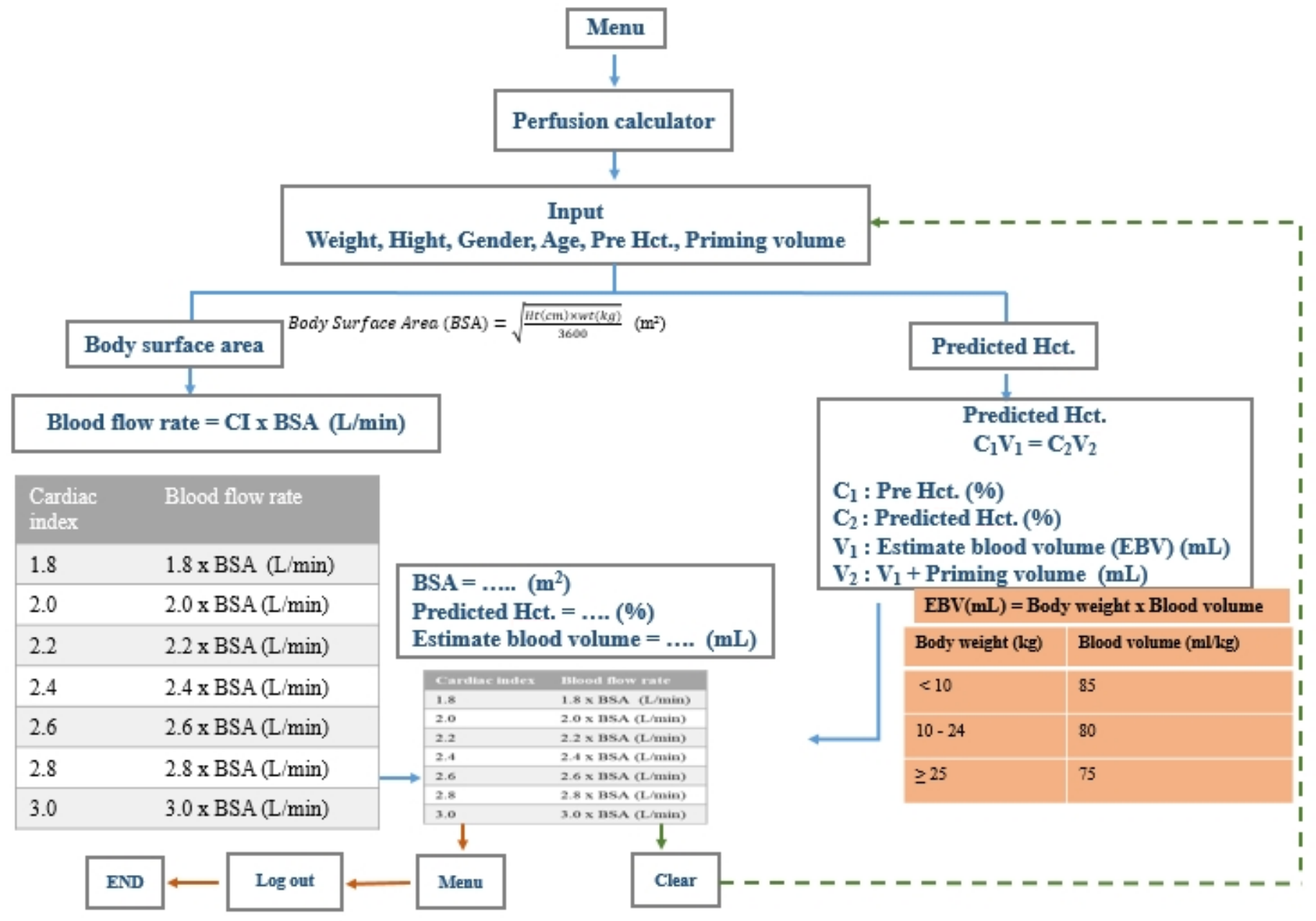

Figure 4

Algorithm of Perfusion Calculator 